\title{
Dietary Quality and Comorbidities of Adults at Medical Risk for Diabetes
}

\author{
Joan A. Vaccaro, Daniela Safina, Lauren Grunspan, Fatma G. Huffman* \\ Department of Dietetics and Nutrition, Florida International University, Miami, USA \\ Email: "huffmanf@fiu.edu
}

Received 2 February 2014; revised 4 March 2014; accepted 16 March 2014

Copyright (C) 2014 by authors and Scientific Research Publishing Inc.

This work is licensed under the Creative Commons Attribution International License (CC BY).

http://creativecommons.org/licenses/by/4.0/

(c) (i) Open Access

\begin{abstract}
Background and aims: The current study evaluates following a special diet with diet quality and comorbidities (hypertension, hypercholesterolemia, and obesity) in four racial/ethnic groups diagnosed with prediabetes or "at risk for diabetes". Methods and results: This is a cross-sectional analysis of data from the National Health and Nutrition Examination Surveys (NHANES), 20072008 and 2009-2010. Sample weights were used to achieve a representative sample. Data were available for $N=2666$ adults, aged $\geq 20$ years (508 Mexican American, 294, Other Hispanic, 616 Black non-Hispanic, and 1248 White non-Hispanic) who were medically diagnosed with either prediabetes or "at risk for diabetes". Those reporting following a special diet had greater odds of meeting saturated fat guidelines $(<\mathbf{1 0 \%}$ of calories) but no greater odds of adequate fiber (14 $\mathrm{g} / 4184 \mathrm{~kJ}(1000 \mathrm{Kcal}))$ as compared to those not following a special diet. There was a significant association of following a diet by number of comorbidities. Regardless of race/ethnicity, reporting following a special diet was more likely for those having more comorbidities; however, number of comorbidities was not associated with diet quality. There were racial/ethnic differences in following a special diet and its association with adequate fiber intake for persons having health risks for diabetes. Black non-Hispanics who did not follow a special diet had lower odds of meeting fiber intake requirements. Conclusion: Low adherence $(<15 \%)$ to fiber dietary guidelines was found across race/ethnicity for adults diagnosed at health risk for diabetes.
\end{abstract}

\section{Keywords}

Prediabetes; Dietary Quality; Hypercholesterolemia; Obesity; Hypertension

\footnotetext{
Corresponding author.

The authors of this paper published an Erratum in OJPM Vol. 4, No. 6 to correct some mistakes in this article. http://dx.doi.org/10.4236/ojpm.2014.46060
} 


\section{Introduction}

The incidence of type 2 diabetes, approximately 95\% of diabetes cases, has risen in epidemic proportions in the United States with 1.9 million new cases of diabetes diagnosed in people aged 20 years and older in 2010 [1]. Hispanics and Black non-Hispanics have nearly twice the prevalence of diabetes as compared to White nonHispanics [1]. Targeting persons at risk for diabetes may be an effective point of intervention for preventing or delaying the onset of type 2 diabetes. According to the Center for Disease Control and Prevention (CDC) 35\% of US adults aged 20 years or older had prediabetes, based on fasted blood glucose or glycated hemoglobin levels above normal, but below the cutoff for diabetes [1]. Prediabetes has been associated with elevated risk for cardiovascular disease [2] [3].

In addition to individuals diagnosed with prediabetes, individuals with a medical diagnosis of "at risk for diabetes" may have normal glucose levels but have other risk factors which place them at a greater risk for diabetes as compared to the general population [4]. Classification of "at risk for diabetes" may include having comorbidities such as hypertension, obesity, and dyslipidemia (low HDL, high triglycerides), other risk factors (advanced age, physical inactivity, family history of diabetes, hypercholesterolemia (high atherogenic lipoproteins), cardiovascular disease, or belonging to certain race/ethnicity) [4]. Individuals with any of the metabolic risk factors for diabetes, if left untreated, are more likely to suffer a poorer quality of life and premature mortality as compared to individuals without cardiovascular disease.

Changing dietary behavior is an established component to prevent or retard the onset of type 2 diabetes for adults diagnosed with either pre-diabetes or at risk for diabetes in several major interventions in the US [5] [6], Europe [7] and China [8]. Although there is no consensus on the composition of macronutrients for diabetes prevention, weight loss and increasing physical activity are recommended [4]. The Harvard School of Public Health recommends specific dietary changes for individuals with pre-diabetes or at risk for diabetes which include adequate dietary fiber and lower saturated fat intake as a method of weight control [9]. As a means to reduce cardiovascular diseases, the reduction of saturated fat to less than $10 \%$ of daily calories is a recommendation by the US Dietary Guidelines [10] and the American Heart Association [11]. Low saturated fat and adequate fiber intakes are key recommendations for persons with diabetes [4].

The aim of the present analysis was to evaluate the level of following a special diet with dietary behavior, comorbidities, and sociodemographic characteristics of adults who were diagnosed with pre-diabetes or "at risk for diabetes". It was hypothesized that those who met daily requirements for fiber and saturated fat intakes would be more-likely to report following a special diet. Furthermore, we hypothesized that those with related comorbidities: hypertension, hypercholesterolemia, and obesity would be more-likely to report following a special diet as compared to their counterparts. Although prediabetes is more commonly associated with dyslipidemia (elevated triglycerides and low HDL-cholesterol) than hypercholesterolemia, the comorbidities assessed in this study represent independent risk factors for coronary heart disease.

\section{Methods}

\subsection{Sample}

The data used in this study were approved by the research ethics board and publically available from appending two cycles from the National Health and Nutrition Examination Surveys (NHANES), 2007-2008 and 2009-2010 [12]. The study was conducted according to the guidelines laid down in the Declaration of Helsinki and all procedures involving human subjects were approved by the National Center for Health Statistics Research Ethics Review Board. NHANES uses a complex, multistage, probability sample design to obtain representative samples of the noninstitutionalized, civilian US population. All data used for this study were available for public use and approved by the research ethics board. More information concerning data collection methods is available at the website: http://www.cdc.gov/nchs/nhanes.htm. This study acquired data from male and female adults $\geq 20$ years of age $(N=11,577)$. The final sample contained adults, $\geq 20$ years, medically diagnosed with prediabetes or "at risk for diabetes", belonging to the category Mexican American (MA), Other Hispanic (OH), Black, nonHispanic (BNH), or White non-Hispanic (WNH) with an energy intake between 2092 and $20920 \mathrm{~kJ}$ (500 and $5000 \mathrm{Kcal}$ ) per day who responded either yes or no to following a special diet and who reported "yes" to either being told they had prediabetes or a health risk for diabetes. Data were available for $n=2666$ adults (508 MA, 294, OH, $616 \mathrm{BNH}$, and $1248 \mathrm{WNH}$ ). 


\subsection{Study Variables}

Following a special diet was recoded as yes or no. The original variable has a category for "not sure". Since there was less than $0.3 \%$ who reported not sure they were coded as missing. Classification with prediabetes or health risk for diabetes was based on answering yes to either question: "health professional told you that you had pre-diabetes" or "health professional told you that you were at risk for diabetes". Diet quality was assessed as having adequate fiber $14 \mathrm{~g} / 4184 \mathrm{~kJ}(1000 \mathrm{Kcal})$ and consuming $<10 \%$ of calories from saturated fats. Diet variables were based on an in-person, 24-hour recall interview at the Mobile Examination Center (MEC). Participants were asked questions on the types, amounts, and preparation of all foods and beverages consumed within a 24-hour period prior to the interview. The second day, 24-hour recall, conducted by telephone interview, was not used due to missing values. Health insurance was coded yes for those who responded to having health insurance in the past 12 months. Education was collapsed into three categories: not completing high school, receiving a high school degree or GED, and completing at least some college. Smoking was classified as either currently smoking or not. Waist circumference was used as a continuous variable. Body mass index (BMI) was classified into four categories <25, $25-29.9,30-34.9$ and $\geq 35$. A variable was made for obesity using a BMI of 30 or more as the cutoff for obesity. Hypercholesterolemia was based on the National Cholesterol Education Program's (NCEP-ATP III) guideline of high non-HDL cholesterol ( $\geq 130 \mathrm{mg} / \mathrm{dL}$ ) [13] and the American Association of Clinical Endocrinologists' (AACE) guideline of $30 \mathrm{mg} / \mathrm{dL}$ above the LDL-cholesterol goal (100 $\mathrm{mg} / \mathrm{dL}$ ) [14]. Hypertension was considered present if systolic blood pressure $\geq 140 \mathrm{~mm} \mathrm{Hg}$ or diastolic blood pressure $\geq 90 \mathrm{~mm} \mathrm{Hg}$ based on the National Institutes of Medicine, National Blood, Lung, and Heart Institute's Seventh Report of the Joint National Committee [15] or currently taking anti-hypertension medication.

\subsection{Statistical Analyses}

Prior to analysis continuous variables were tested for normality by the Kolmogorov-Smirnov test. Variables not normally distributed were transformed and re-tested. Variables that were unable to be normalized were categorized. Cross-tabulation for complex samples with the Pearson's $\chi^{2}$ was used to assess group differences in the bivariate study variables by race/ethnicity. Logistic regression analysis for complex samples was conducted to measure the adjusted odds ratio with 95\% confidence intervals of following a special diet for adults with prediabetes. The dependent variable was following a special diet which was dichotomized as yes or no. The covariates included comorbidities (obesity, hypertension, and hypercholesterolemia), diet quality (adequate fiber and recommended saturated fat) and sociodemographics (race/ethnicity, gender, age, education, currently smoking, and health insurance). Main and two-way interactions for race/ethnicity and gender were tested. The model fit was determined by the classification table. Analyses were performed with the complex sample analysis module of SPSS version 20 statistical software package. Adjusted sample weights were used to account for the multistage stratified cluster sampling design and to account for the unequal probabilities of selection, and nonresponse. The sample weights used were based on the data file with the smallest sample size, the Mobile Examination Center (MEC), and were computed using the average of the 2-year sample weights for each cycle, as per guidelines set by NHANES [16]. A p-value of $<0.05$ was considered significant.

\section{Results}

For this population who are at risk for diabetes or have prediabetes, the comorbidities and diet quality by race/ ethnicity are shown in Table 1. BNH had the highest prevalence of hypercholesterolemia, followed by WNH; whereas MA and $\mathrm{OH}$ had the lowest prevalence. Both WNH and BNH had a higher prevalence of hypertension as compared to Hispanics. Black non-Hispanics and MA had higher rates of obesity as compared to $\mathrm{OH}$ and WNH. Between 10\% - 14\% reported following a special diet. Meeting adequate fiber intake was low in all racial/ethnic groups ( $7 \%$ - 15\%). There were no differences in following a special diet by race/ethnicity; however, more WNH followed by BNH consumed higher than recommended saturated fat as compared to Hispanics and BNH had the lowest percent of adequate fiber compared to other racial/ethnic groups.

Diets selected were either weight-loss/low calorie (52.1\%); low-fat/low cholesterol (19.1\%); low salt/low sodium (17.1\%); low carbohydrate (2.9\%); sugar-free/low sugar (2.0\%); weight gain/muscle building (1.5\%); diabetes $(1.5 \%)$; high fiber $(0.9 \%)$; high protein $(0.3 \%)$ or other special diet $(3.8 \%)$. There were no responses in the low fiber diet. There was a significant association of following a diet by number of comorbidities as shown 
Table 1. Comorbidities and diet quality by race/ethnicity of the population with prediabetes or "at risk for diabetes”.

\begin{tabular}{cccccc}
\hline Variable & MA & OH & BNH & WNH & P \\
\hline $\begin{array}{c}\text { Hypercholesterolemia } \\
\text { Non-HDL-C } \geq 130 \mathrm{mg} / \mathrm{dL}\end{array}$ & $24.5^{\mathrm{a}}(20.4,29.2)$ & $27.6^{\mathrm{a}, \mathrm{b}}(21.6,34.4)$ & $37.1^{\mathrm{c}}(32.5,41.8)$ & $29.9^{\mathrm{b}}(27.6,32.3)$ & 0.002 \\
$\begin{array}{c}\text { Hypertension SBP } \geq 140 ; \\
\text { DBP } \geq 90(\mathrm{~mm} \mathrm{Hg})\end{array}$ & $27.0^{\mathrm{a}}(23.2,31.3)$ & $31.3^{\mathrm{a}}(23.3,40.6)$ & $44.4^{\mathrm{b}}(40.2,48.6)$ & $45.5^{\mathrm{b}}(42.3,48.7)$ & $<0.001$ \\
Obesity BMI $\geq 30 \mathrm{~kg} / \mathrm{m}^{2}$ & $52.8^{\mathrm{a}}(49.0,56.6)$ & $47.4^{\mathrm{a}, \mathrm{b}}(40.7,54.2)$ & $53.3^{\mathrm{a}}(49.2,57.3)$ & $42.6^{\mathrm{b}}(39.1,46.1)$ & $<0.001$ \\
Following a special diet (yes) & $10.5(8.3,13.2)$ & $13.8(10.9,17.4)$ & $10.5(8.3,13.3)$ & $12.8(11.2,14.6)$ & 0.156 \\
$\begin{array}{c}\text { SF }<10 \% \text { of calories } \\
\text { Adequate fiber intake } \geq 14 \mathrm{~g} / 4184 \mathrm{~kJ} \\
(\mathrm{~g} / 1000 \mathrm{Kcal})\end{array}$ & $55.5^{\mathrm{a}}(50.4,60.6)$ & $60.2^{\mathrm{a}}(53.2,66.7)$ & $48.3^{\mathrm{b}}(43.8,52.9)$ & $36.8^{\mathrm{c}}(33.6,40.1)$ & $<0.001$ \\
& $14.6^{\mathrm{a}}(11.7,18.1)$ & $10.6^{\mathrm{a}, \mathrm{b}}(7.6,14.5)$ & $6.9^{\mathrm{c}}(5.0,9.4)$ & $11.0^{\mathrm{b}}(8.8,13.6)$ & 0.014 \\
\hline
\end{tabular}

Abbreviations: MA = Mexican Americans; OH = Other Hispanics; BNH = Black, non-Hispanic; WNH = White, non-Hispanic; BMI = body mass index; SF = saturated fat intake. Notes: Estimate is percent ( $95^{\text {th }}$ confidence intervals). Each superscript letter denotes a subset of race/ethnicity categories whose column estimates do not differ significantly from each other.

in Table 2. A greater percent of those with two or three comorbidities reported following a special diet as compared to those with one or no comorbidities. The results of the hypothesis test are depicted in Table 3 . Those with two or three comorbidities had a greater odds of reporting following a special diet than those with one or no comorbidities [OR $=1.85(1.25,2.74), \mathrm{P}=0.003$ ]. Individuals who consumed less than 10 percent of their calories from saturated fat were also more likely to report following a special diet $[\mathrm{OR}=1.40(1.01,1.96)$; $\mathrm{P}=$ 0.046]. Higher odds of following a special diet was marginally associated with adequate fiber intake; albeit the relationship was not significant $[\mathrm{OR}=1.42(0.98,2.07), \mathrm{P}=0.062]$. Cross-tabulation of comorbidity number with adequate fiber and saturated fat showed no significant association $(\mathrm{P}=0.118 ; \mathrm{P}=0.713$, respectively). A reduced model adjusting for age and gender was run to test the main and 2-way interactions of race/ethnicity and following a diet for the odds of adequate fiber as presented in Table 4. There were differences by race/ethnicity by level of following a special diet with adequate fiber intake. Racial/ethnic differences were only significant for those reporting not following a diet. MA reporting not following a diet had a higher odd of consuming adequate fiber as compared to WNH; whereas, BNH not following a diet had a lower odds of meeting adequate fiber intake as compared to WNH.

\section{Discussion}

Increasing fiber and lowering saturated fat intake are the main dietary objectives of a prescribed diet for diabetes prevention. Although we found no racial/ethnic differences in reporting following a special diet in those diagnosed with prediabetes or "at risk for diabetes" there were significant differences by race/ethnicity in self-reported dietary intake. Inadequate fiber intake was more likely for BNH as compared to other racial/ethnic groups in this study. Foods higher in fiber such as whole grains, fruits and vegetables, may be more challenging to incorporate into a diet for individuals based on their circumstances. Even though environmental, economic and personal barriers for lifestyle change exist across race/ethnicity, these barriers may be greater for minorities with limited resources [17].

Regardless of race/ethnicity, reporting following a special diet was more likely for those having more comorbidities; yet, number of comorbidities was not associated with adequate fiber or meeting the guidelines for saturated fat intake. Data were not available as to the reasons for following a diet. Increased likelihood to follow a diet for persons who have comorbidities may be due to multiple providers prescribing a diet and/or greater perceived susceptibility toward developing diabetes.

To date, there are no studies comparing number of comorbidities in persons at risk for diabetes and dietary adherence. Since individuals diagnosed with prediabetes or "at risk for diabetes" have been advised of the threat of the disease, having concurrent chronic conditions such as hypertension, hypercholesterolemia, or obesity could increase individuals' perceived susceptibility to cardiovascular disease, hence increase their likelihood for dietary changes. These concepts are congruent with the Health Belief Model for preventive behavior [18].

Although reducing saturated fat from the diet was more likely for persons who reported following a special 
Table 2. Number of comorbidities and following a special diet.

\begin{tabular}{ccc}
\hline $\begin{array}{c}\text { Number of } \\
\text { comorbidities }\end{array}$ & $\begin{array}{c}\text { Percent }\left(95^{\text {th }} \mathrm{CI}\right) \text { responding “yes” } \\
\text { for following a special diet }\end{array}$ & SE (\%) \\
\hline All three & $16.3(11.0,23.5)$ & 3.0 \\
Two & $16.4(13.2,20.1)$ & 1.7 \\
One & $9.7(8.0,11.7)$ & 0.9 \\
None & $10.6(8.1,13.7)$ & 1.4 \\
\hline
\end{tabular}

"Morbidities included hypertension, hypercholesterolemia, or obesity. Model: Comorbidity by special diet; Pearson's Chi Square = 22.3; $\mathrm{P}=0.002$.

Table 3. Odds ratio of sociodemographics, diet quality and comorbidities with following a special diet.

\begin{tabular}{ccc}
\hline Variable & OR $(95 \%$ CI $)$ & P \\
\hline Race/ethnicity & - & 0.277 \\
Age (years) & $0.99(0.98,1.0)$ & 0.033 \\
Female & $2.20(1.63,2.97)$ & $<0.001$ \\
Currently smoking (yes) & $0.82(0.55,1.22)$ & 0.316 \\
Education level & - & 0.167 \\
Health insurance (yes) & $1.45(1.02,2.04)$ & 0.037 \\
Adequate fiber ( $\geq 14$ g/1000 Kcal) & $1.42(0.98,2.07)$ & 0.062 \\
Adequate saturated fat intake $<10 \%$ of calories & $1.40(1.01,1.96)$ & 0.046 \\
Two or three comorbidities vs one or none & $1.85(1.25,2.74)$ & 0.003 \\
One or no comorbidities (reference) & 1.00 & \\
\hline
\end{tabular}

Table 4. Odds ratio of following a special diet and race with having adequate fiber intake.

\begin{tabular}{ccc}
\hline Variable & OR $(95 \%$ CI $)$ & P \\
\hline Special diet (yes) & $1.46(0.95,2.23)$ & 0.003 \\
Race/ethnicity by special diet $^{*}$ & - & 0.038 \\
MA—yes & $1.37(0.60,3.14)$ & 0.447 \\
MA—no & $1.99(1.19,3.35)$ & 0.011 \\
OH—yes & $1.68(0.58,4.94)$ & 0.332 \\
OH—no & $1.12(0.70,1.81)$ & 0.672 \\
BNH—yes & $1.96(0.62,6.21)$ & 0.242 \\
BNH—no & $0.58(0.35,0.97)$ & 0.037 \\
WNH (reference) & 1.00 & - \\
Race/ethnicity & 1.00 & 0.190 \\
Age (years) & $1.02(1.01,1.03)$ & 0.001 \\
Males & $0.56(0.40,0.76)$ & 0.001 \\
\hline
\end{tabular}

*Yes refers to following a special diet and no refers to not following a special diet. Abbreviations: $\mathrm{MA}=$ Mexican American, $\mathrm{OH}=$ Other Hispanic; $\mathrm{BNH}=$ Black non-Hispanic; WHN = White non-Hispanic.

diet, the relationship of diet and adequate fiber intake was not significant in this study. Our results parallel Hendrychova et al. [19] who found adherence to fat-related dietary behavior to be easier to follow as compared to fiber-related dietary behaviors for outpatients with type 2 diabetes. In contrast, Absetz et al. [20] reported improvement in meeting fiber intake, but not fat consumption recommendations in patients at high risk for diabetes from 16 health care centers after a lifestyle intervention. Resistance to dietary change for both fat and fiber was 
reported as a barrier with no significant differences between the intervention (lifestyle management through motivational interviewing) and usual care groups of patients at high risk for diabetes [21]. Over half of both groups reported they already met the standards for dietary fiber, total fat, and physical activity [21]. We could not ascertain whether those following a diet made changes because of intense counselling or because they received printed guidelines; however, Lakerveld and colleagues [22] concluded that there were no differences in dietary behavior in a randomized control trial comparing an intense lifestyle intervention with a control group who received health brochures for a population at risk for diabetes. Cardona-Morrell et al. [23] found self-reported dietary and physical activity measures as clinical indicators of effectiveness of lifestyle interventions in preventing or delaying diabetes were scarce, based on a systematic review. The authors indicated that of these 12 studies, reduction in percent calories from total fat and increase in fiber intake per day were the chosen dietary outcome measures in three studies and the others did not report any dietary measures [23].

\section{Strengths and Limitations}

This study has strengths and limitations. One of the strengths of our study was the use of a nationally representative sample of US adults. Another benefit of this study was the current comparison of minorities to WHN in areas of cardiometabolic risk factors. Although efforts have been made to reduce health disparities, prevalence of health outcomes by race/ethnicity needs to be assessed every few years. There were several limitations to this study. It was not specified whether a health care professional prescribed the diet. A potential limitation of this was combining all categories of special diet for this study population; however, for this study no persons in the sample selected the low-fiber diet. It is questionable whether weight-gaining or high-protein diets would be prescribed for persons with prediabetes. There was an option for "other special diet" which was not specified. Individuals at risk for diabetes may have chosen the Mediterranean diet, Harvard Healthy Eating Plate, MyPlate, or alternative health diets such as anti-cancer, vegetarian or vegan.

In summary, there was a significant, positive association of following a diet by number of comorbidities; however, the association of diet quality and number of comorbidities was not significant. Individuals diagnosed with prediabetes or "at risk for diabetes" and who are reported they are following a special diet have greater odds of meeting the recommendation for saturated fat; however, they are not any more likely to consume adequate fiber than those not following a special diet. Diet adherence to fiber for this population at risk for diabetes was least likely for BNH as compared to other racial/ethnic groups. Since diet quality is a key component to preventing diabetes for those at medical risk and managing comorbidities such as hypertension, hypercholesterolemia, and obesity, measures should be taken by health care professionals to involve public health nutritionists in the treatment plan. Our study reinforces the need for public health nutritionist to act as a modifying force by increasing the action of dietary adherence specific to race/ethnicity for individuals at risk for diabetes.

\section{Acknowledgments}

Source of funding: Data collected were available to the public by NHANES. Conflicts of interest: The authors declare that they have no conflicts of interest. Authors' contributions: All authors developed the idea of the manuscript and designed the study. JAV drafted and wrote the manuscript. JAV was responsible for data analysis. FGH, DS, and LG critically reviewed the helped revise the manuscript. All authors read and approved the final manuscript.

\section{References}

[1] Centers for Disease Control and Prevention (2011) National Diabetes Fact Sheet: National Estimates and General Information on Diabetes and Prediabetes in the United States, 2011. US Department of Health and Human Services, Centers for Disease Control and Prevention, Atlanta.

[2] DeFronzo, R.A. and Abdul-Ghani, M. (2011) Assessment and Treatment of Cardiovascular Risk in Prediabetes: Impaired Glucose Tolerance and Impaired Fasting Glucose. American Journal of Cardiology, 108, 3B-24B. http://dx.doi.org/10.1016/j.amjcard.2011.03.013

[3] Nonogaki, K. (2012) Dysglycemia and Cardiovascular Risk. Journal of the American College of Cardiology, 60, 1121.

[4] American Diabetes Association (2013) Standards of Medical Care in Diabetes_-2013. Diabetes Care, 36, S11-S66. http://dx.doi.org/10.2337/dc13-S011

[5] Knowler, W.C., Barrett-Connor, E., Fowler, S.E., et al. (Diabetes Prevention Research Group) (2002) Reductions in 
the Incidence of Type 2 Diabetes with Lifestyle Intervention or Metformin. The New England Journal of Medicine, 346, 393-403. http://dx.doi.org/10.1056/NEJMoa012512

[6] Knowler, W.C., Fowler, S.E., Hamman, R.F., et al. (2009) 10-Year Follow-Up of Diabetes Incidence and Weight Loss in the Diabetes Prevention Program Outcomes Study. Lancet, 374, 1677-1686. http://dx.doi.org/10.1016/S0140-6736(09)61457-4

[7] Jaakko Tuomilehto, J., Lindström, J., Eriksson, J.G., et al. (2001) Prevention of Type 2 Diabetes Mellitus by Changes in Lifestyle among Subjects with Impaired Glucose Tolerance. The New England Journal of Medicine, 344, 343-1350. http://dx.doi.org/10.1056/NEJM200105033441801

[8] Pan, X.R., Li, G.W., Hu, Y.H., et al. (1997) Effects of Diet and Exercise in Preventing NIDDM in People with Impaired Glucose Tolerance: The Da Qing IGT and Diabetes Study. Diabetes Care, 20, 537-544. http://dx.doi.org/10.2337/diacare.20.4.537

[9] Harvard School of Public Health (2013) Simple Steps to Preventing Diabetes. http://www.hsph.harvard.edu/nutritionsource/preventing-diabetes-full-story/

[10] US Department of Agriculture and US Department of Health and Human Services (2010) Dietary Guidelines for Americans. US Government Printing Office, Washington DC.

[11] American Heart Association Nutrition Committee, Lichtenstein, A.H., Appel, L.J., Brands, M., et al. (2006) Diet and Lifestyle Recommendations Revision 2006: A Scientific Statement from the American Heart Association Nutrition Committee. Circulation, 114, 82-96. http://dx.doi.org/10.1161/CIRCULATIONAHA.106.176158

[12] National Health and Nutrition Examination Survey (2013) Questionnaires, Datasets and Related Documentation. http://www.cdc.gov/nchs/nhanes/nhanes questionnaires.htm

[13] National Cholesterol Education Program (NCEP) ATP III. US Department of Health and Human Services (2013) Public Health Service National Institutes of Health National Heart, Lung and Blood Institute NIH 2012. Publication No. 01-3305. https://www.nhlbi.nih.gov/guidelines/hypertension/

[14] Jellinger, P.S., Smith, D.A., Mehta, A.E., et al. (2012) American Association of Clinical Endocrinologists (AACE) Guidelines for the Management of Dyslipidemia and Prevention of Atherosclerosis. Endocrine Practice, 18, 1-78. http://dx.doi.org/10.4158/EP.18.S1.1

[15] (2013) Seventh Report of the Joint National Committee on Prevention, Detection, Evaluation, and Treatment of High Blood Pressure (JNC 7). NIH Publication No. 03-5231. http://www.nhlbi.nih.gov/guidelines/hypertension/phycard.pdf

[16] (2013) National Health and Nutrition Examination Survey (NHANES): Analytical and Reporting Guidelines, 20112012. National Center for Health Statistics (NCHS): Center for Disease Control and Prevention, Hyattsville. http://www.cdc.gov/nchs/data/nhanes/analytic_guidelines_11_12.pdf

[17] Orzech, K.M., Vivian, J., Torres, M.A., Armin, J. and Shaw, S.J. (2013) Diet and Exercise Adherence and Practices among Medically Underserved Patients with Chronic Disease: Variation across Four Ethnic Groups. Health Education \& Behavior, 40, 56-66. http://dx.doi.org/10.1177/1090198112436970

[18] Jan, N.K. and Becker, M.H. (1984) The Health Model a Decade Later. Health Education Quarterly, 11, 1-47. http://dx.doi.org/10.1177/109019818401100101

[19] Hendrychova, T., Vytrisalov, M., Vlcek, J., Smahelova, A. and Kubena, A.A. (2013) An Analysis of Fat-Related and Fiber-Related Behavior in Men and Women with Type 2 Diabetes Mellitus: Key Findings for Clinical Practice. Patient Prefer Adherence, 7, 877-884. http://dx.doi.org/10.2147/PPA.S47497

[20] Absetz, P., Valve, R., Oldenburg, B., Heinonen, H., Nissinen, A., Fogelhom, M., Llvesmaki, V., Taljia, M. and Uutell, A. (2007) Type 2 Diabetes Prevention in the "Real World." One Year Results of the GOAL Implementation Trial. Diabetes Care, 30, 2465-2470. http://dx.doi.org/10.2337/dc07-0171

[21] Vermunt, P.W.A., Milder, I.E., Wielaard, F., Baan, C.A., Schelfhout, J.D.M., Westert, G.P. and van Oers, H.A.M. (2013) Behavior Change in a Lifestyle Intervention for Type 2 Diabetes Prevention in Dutch Primary Care: Opportunities for Intervention Content. BMC Family Practice, 14, 78. http://dx.doi.org/10.1186/1471-2296-14-78

[22] Lakerveld, J., Bot, S.D., Chinapaw, M.J., van Tlder, M.W., Kostense, P.J., Dekker, J.M. and Nijpels, G. (2013) Motivational Interviewing and Problem Solving Treatment to Reduce Type 2 Diabetes and Cardiovascular Disease Risk in Real Life: A Randomized Controlled Trial. International Journal of Behavioral Nutrition and Physical Activity, $10,47$. http://www.ijbnpa.org/content/10/1/47

[23] Cardona-Morrell, M., Rychetnik, L., Morrell, S.L., Espinel, P.T. and Bauman, A. (2010) Reduction of Diabetes Risk in Routine Clinical Practice: Are Physical Activity and Nutrition Interventions Feasible and Are the Outcomes from Reference Trials Replicable? A Systematic Review and Meta-Analysis. BMC Public Health, 10, 653. http://dx.doi.org/10.1186/1471-2458-10-653 